\title{
Logics for approximate and strong entailments*
}

\author{
Francesc Esteva ${ }^{1}$, Lluís Godo ${ }^{1}$, \\ Ricardo O. Rodríguez ${ }^{2}$, Thomas Vetterlein ${ }^{3}$ \\ ${ }^{1}$ IIIA - CSIC, 08193 Bellaterra, Spain; \\ \{esteva, godo\}@iiia.csic.es \\ ${ }^{2}$ University of Buenos Aires, 1428 Buenos Aires, Argentina; \\ ricardoddc.uba.ar \\ ${ }^{3}$ Johannes Kepler University Linz, Altenberger Straße 69, 4040 Linz, Austria; \\ Thomas.Vetterlein@jku.at
}

\begin{abstract}
We consider two kinds of similarity-based reasoning and formalise them in a logical setting. In one case, we are led by the principle that conclusions can be drawn even if they are only approximately correct. This leads to a graded approximate entailment, which is weaker than classical entailment. In the other case, we follow the principle that conclusions must remain correct even if the assumptions are slightly changed. This leads to a notion of a graded strong entailment, which is stronger than classical entailment. We develop two logical calculi based on the notions of approximate and of strong entailment, respectively.
\end{abstract}

\section{Introduction}

Reasoning under practical circumstances is often inexact. Assumptions might be fulfilled only in an approximate way but conclusions are drawn anyway. Different epistemic aspects may be involved, like uncertainty, preference or similarity. In order to formalise such kind of reasoning we need to go beyond classical propositional logic. The present paper deals with similarity-based reasoning. The work can be seen in the more general framework of reasoning by analogy and has applications, for example, in classification, case-based reasoning, or interpolation.

${ }^{*}$ This paper is a fully revised and extended version of the conference paper [4], presented at the 15th Spanish Congress on Technologies and Fuzzy Logic (ESTYLF), Huelva, Spain, Febr. 4-5, 2010. 
In the setting of fuzzy set theory, it was E. Ruspini who initiated the issue of similarity-based reasoning in [12], where he defines a semantics for fuzzy sets based on fuzzy similarity relations. Following his approach, the topic of similaritybased reasoning has been worked out in a series of papers from a logical perspective $[2,3,11,6]$. In these papers the authors define and study the so-called approximate entailment. Here, we consider in addition the so-called strong entailment, which is in a sense dual to approximate entailment.

Similarity-based inference has been treated in a logical setting and also the present paper takes up this approach. Our approach is conceptually closely related to the work presented in $[8,7]$. The logics of metric spaces defined there are conceived as knowledge representation formalisms incorporating the notion of a distance, which is a dual to the notion of similarity considered here. A further approach to similarity-based approximate reasoning, guided by somewhat different ideas, is due to Ying [13].

The logics considered here formalise the effect of small changes on the validity of logical relationships. Consider a pair of propositions such that none is implied by the other one; we may then still ask if one of these two propositions is a consequence of the other one by means of a slight change. Conversely, for a pair of propositions one of which is a consequence of the other one, we may ask if this consequence relation is stable under small changes.

Indeed, a rule like

$$
\text { if } \varphi \text { then } \psi \text {, }
$$

understood as the logical implication of classical propositions, can be modified in the presence of a similarity relation in at least two different ways:

$$
\begin{aligned}
& \text { if } \varphi \text { then approximately } \psi \\
& \text { or } \\
& \text { if approximately } \varphi \text { then } \psi
\end{aligned}
$$

The possibilities offered by the usual, that is, classical, propositional logic are certainly insufficient here. Indeed, when considering relationships among propositions in classical logic, semantic similarity among propositions does not play a role. It may happen that some conclusion can be drawn from certain assumptions but that conclusion is no longer possible if the assumptions change just slightly.

To formalise inferences as shown above, we have to make precise what we mean by "approximately". We will follow a quantitative approach and use fuzzy similarities relations to this end. Assume that we model a variety of possible situations by a 
set of yes-no propositions. Each proposition represents a set of situations, namely those situations in which it holds. It is then often the case that we may say that certain propositions are close to each other, whereas other propositions differ from each other to a large extent. Accordingly, we will assume that the set of propositions used to characterise a set of varying situations is endowed, in some natural way, with a similarity relation.

Let us illustrate the relevance of our work with two examples. Assume that, in medical decision support, we want to formally represent the fact that a certain laboratory test result implies the presence of a certain disease. More specifically, assume that the following, somewhat idealised, information is given:

(R) If the blood value $\mathrm{BV}$ is equal to or larger than 500 units then the disease $\delta$ is present.

Assume that the rule (R) is to be implemented in a decision support system. As for our formal model, let us take a set of worlds $W$ such that to each $w \in W$ a real number $b$ in the possible range of $\mathrm{BV}$ is assigned as well as the information that $\delta$ is present or not present. The assumption " $\mathrm{BV} \geq 500$ " and the conclusion " $\delta$ is present" then correspond to two subsets of $W$.

Imagine first that reasoning is done by means of classical propositional logic. Assuming $(\mathrm{R})$ to hold then implies that the former subset is contained in the latter; the worlds assigned a value $\mathrm{BV} \geq 500$ and " $\delta$ is not present" are excluded. Thus, a test result "500 units" would lead to the output " $\delta$ is present", but if the result was "499 units" no information would be provided.

In practice, however, $(\mathrm{R})$ would clearly be understood differently. If $\mathrm{BV}$ is just slightly below 500 units, $\delta$ would surely be seriously considered as well. However, the smaller BV is, the less $\delta$ would be found likely. A possibility to formalise this kind of reasoning is offered by the Logic of Approximate Entailment (LAE) discussed below [11]. Namely, define two BV values $b_{1}$ and $b_{2}$ to be similar to the degree, say, $\max \left\{1-\frac{\left|b_{1}-b_{2}\right|}{50}, 0\right\}$. Then, by the so-called approximate entailment, from a BV value of 499 we could conclude "BV $\geq 500$ units" attached with a weight expressing how well this condition is fulfilled. The weight is taken as the degree of similarity between the statement "BV $\geq 500$ units" and the world in which BV is assigned the value 499 , that is, 0.98 . This weight can be then propagated to the conclusion " $\delta$ is present", witnessing that our reasoning is based only on an approximately correct premise.

Next consider an example of a strong entailment. Assume the test result gives a $\mathrm{BV}$ value of 530 units. In this case, we can draw the conclusion that the disease $\delta$ is present without any doubt since the observed BV value satisfies the premise of the 
rule. Moreover, the conclusion will still be valid if we happen to know that the test is not fully reliable and may provide inaccurate values, and hence that the real $B V$ value may be actually somewhat different from 530 . In this setting, we take 0.4 as an indication of the robustness of our inference: 0.4 is the minimal degree $\alpha$ such that any $\mathrm{BV}$ value $v$ similar to 530 to the degree $\geq \alpha$ still fulfils the premise " $\mathrm{BV} \geq$ 500 ". Notice however that, in contrast to the case of approximate entailment, here the smaller this value is, the more robust is the inference of drawing the conclusion " $\delta$ is present". This is the idea of strong entailment put forward in this paper.

Further examples can be found in the field of mathematical morphology; see, e.g., [1]. The so-called dilation and erosion operators play a role analogous to the approximate and strong entailment relations.

The formal framework for similarity-based reasoning which we use here follows the lines of $[2,3,11,6]$. The most flexible approach is provided by multi-modal logics [3]. In this case, the universe of discourse $W$ is equipped with a fuzzy similarity relation and a modal operator $\nabla_{\alpha}$ is added to the language of classical propositional logic for each $\alpha$ from a fixed set $V$ of truth degrees. $\nabla_{\alpha}$ is interpreted by the $\alpha$-neighbourhood operator $U_{\alpha}$ in $W$, i.e. the operator that maps a set of worlds to the bigger set of those worlds that are similar at least to the degree $\alpha$ to some of the original worlds. In the multi-modal framework, the relationships which we consider here can be expressed as follows. In approximate reasoning we deal with conditional statements of the form

$$
\varphi \rightarrow \diamond_{\alpha}(\psi)
$$

which may be read " $\psi$ approximately follows from $\varphi$ to the degree $\alpha$ ". (1) holds if $e(\varphi) \subseteq U_{\alpha}(e(\psi))$, where $e$ assigns to propositions the set of worlds in which they hold. Similarly, in strong reasoning, we deal with conditionals of the form

$$
\diamond_{\alpha}(\varphi) \rightarrow \psi
$$

which may be red "any fact resembling $\varphi$ to the degree $\alpha$ implies $\psi$ ". (2) holds if $U_{\alpha}(e(\varphi)) \subseteq e(\psi)$.

In this paper we consider less expressive logics than those discussed in [3]. In fact we develop two calculi, the Logic of Approximate Entailment and the Logic of Strong Entailment, that just allow to express graded conditional relationships of the form (1) or (2) respectively. Namely, the Logic of Approximate Entailment is defined to reason about graded implications of the form $\varphi>_{\alpha} \psi$ which correspond to (1), while the logic of Strong Entailment deals with reasoning about graded implications $\varphi \succ_{\alpha} \psi$ which correspond to (2). 
In the rest of the paper we proceed as follows. First we specify in Sections 2 and 3 the formal semantic frameworks for approximate and strong entailment respectively, and compile some basic properties.Then, in Section 4 we present the Logic of Approximate Entailment and prove its completeness, while in Section 5 we proceed analogously for the case of the Logic of Strong Entailment. We end up in Section 6 with some remarks about merging both logics into a single one and discuss some of the many remaining challenges for future work.

\section{A graded approximate entailment}

Our setting for approximate reasoning is due to Ruspini [12]. Here we proceed in the style of the papers $[2,3,11,6]$.

The basic framework contains, first of all, a set $W$ of possible worlds. $W$ represents a set of possible situations varying with respect to some selected properties of the objects under consideration. The rule for medical decision support might be a guiding example; here $W$ consists of the different possible combinations of a patient's signs, symptoms, and diseases.

Furthermore, we assume that $W$ is endowed with a similarity relation $S$. In this way every two worlds in $W$ are assigned the degree $\alpha \in[0,1]$ to which they differ; the larger $\alpha$ is the more they resemble. In particular, $S(v, w)=0$ means that $v$ and $w$ are totally unrelated, $S(v, w)=1$ means that $v$ and $w$ coincide. Again referring to the medical example, whenever signs or symptoms are quantifiable, like continuous-valued laboratory test results, a measure of similarity can be defined by means of the differences.

Definition 2.1. Let $W$ be any non-empty set; let $\odot$ be a t-norm; and let $V$ be a subset of the real unit interval containing 0 and 1 and closed under $\odot$. A function $S: W \times W \rightarrow V$ will be called a $\odot$-similarity relation ${ }^{1}$ if, for any $u, v, w \in W$, the following properties are fulfilled:

(sr1) $S(u, u)=1$ (reflexivity)

(sr2) $S(u, v)=1$ implies $u=v$ (separability)

(sr3) $S(u, v)=S(v, u)$ (symmetry)

$($ sr4) $S(u, v) \odot S(v, w) \leq S(u, w)(\odot$-transitivity)

\footnotetext{
${ }^{1}$ In the literature, other names like fuzzy equality, indistinguishability relation, etc. have been used to denote fuzzy relations satisfying $(\operatorname{sr} 1),(\operatorname{sr} 3)$ and $(\operatorname{sr} 4)$.
} 
In such a case, we call $(W, S)$ a similarity space.

For the rest of the paper, we will assume that $\odot$ is any fixed t-norm. Accordingly, we will usually drop the explicit reference to the t-norm under consideration; in particular, we will speak simply about similarity relations.

The value $1 \in V$ applies in the case of coincidence, as expressed by the reflexivity property. Conversely, two worlds with the similarity degree 1 necessarily coincide, as established by separability. Note that if $S$ was supposed to fulfil ( $\mathrm{sr} 1$ ), (sr3), (sr4) only, we could identify points $u, v \in W$ such that $S(u, v)=1 ; S$ is compatible with this identification and the induced function would be a similarity relation. Furthermore, similarity is supposed to be a symmetric notion. Finally, $\odot$-transitivity can be viewed as a triangle inequality.

One may think of a similarity space as a generalised metric space. Namely, let $(W, S)$ be a similarity space and put $D(u, v)=1-S(u, v)$ for $u, v \in W$. Then (sr4) translates to $D(u, w) \leq D(u, v) \oplus D(v, w)$, where $\oplus$ is the t-conorm associated to $\odot$. If $\odot$ is the Eukasiewicz t-norm, then $D$ is indeed a metric, which is bounded by 1 . Furthermore, if $\odot$ is the product t-norm, $D$ is, after a rescaling of its range, a metric with values in $\mathbb{R}^{+} \cup\{\infty\}$.

In this paper, propositions will be modelled classically by sets of possible worlds, that is, by subsets of $W$. Let $A \subseteq W$ model a proposition. We write $w \models A$ to express that the proposition represented by $A$ holds in the world $w$, that is, $w \in A$.

If $B \subseteq W$ represents another proposition, classical implication means

$$
\text { for any } w \in W, \quad w \models A \text { implies } w \models B .
$$

This is the subsethood relation, $A \subseteq B$. To make the intention clear we will however write $A=B$ for (3).

The idea of approximate entailment is to formalise this implicational relationship to hold only approximately. We write $w \models{ }^{\alpha} A$, where $\alpha \in[0,1]$, to express that $w$ is not necessarily a world in which $A$ holds, but there is a world $v$ such that $S(w, v) \geq \alpha$ and $v \models A$. Then, we say that $A$ implies $B$ to the degree $\alpha \in V$ if

$$
\text { for any } w \in W, \quad w \models A \text { implies } w \models{ }^{\alpha} B,
$$

that is, any world in which $A$ holds is $\alpha$-similar to a world in which $B$ holds. We write $A \models^{\alpha} B$ for (4). For $A \subseteq W$, let

$$
U_{\alpha}(A)=\{w \in W: S(w, v) \geq \alpha \text { for some } v \in A\}
$$

be the $\alpha$-neighbourhood of $A$. Note that, in particular, $U_{\alpha}(\emptyset)=\emptyset$. Then $A={ }^{\alpha} B$ is equivalent to

$$
A \subseteq U_{\alpha}(B)
$$


Given $A$ and $B$ one may wonder about the greatest $\alpha \in V$ such that $A \models^{\alpha} B$. To this end we consider Ruspini's first measure of the compatibility of one proposition with another one:

$$
I(B \mid A)=\inf _{v \models A} \sup _{w \models B} S(v, w) .
$$

Then $I(B \mid A)>\alpha$ implies $A=^{\alpha} B$. Under certain additional assumptions like for instance the finiteness of $W$, we have an equivalence.

Lemma 2.2. Assume that $W$ is finite and $B \neq \emptyset$. Then $A \models^{\alpha} B$ if and only if $I(B \mid A) \geq \alpha$.

Proof. We have $I(B \mid A)=\min _{v \in A} \max _{w \in B} S(v, w)$. So $I(B \mid A) \geq \alpha$ iff, for all $v \in A, \max _{w \in B} S(v, w) \geq \alpha$ iff for all $v \in A$ there is a $w \in B$ such that $S(v, w) \geq \alpha$. The last statement is, by definition, equivalent to $A={ }^{\alpha} B$.

We compile the basic properties of the approximate entailment relation $\models^{\alpha}$ shown in [2]. Although the relations are among subsets, we will use here and in the next section the logical symbols $\wedge, \vee, \neg$ to denote intersection, disjunction and complementation, respectively.

Lemma 2.3. Let $(W, S)$ be a similarity space. Then the following holds for any $A, B, C \subseteq W$ and $\alpha, \beta \in V$ :

(i) $A \models{ }^{1} B$ if and only if $A \models B$; in particular $A \models^{1} A$ (reflexivity).

(ii) $A \models{ }^{0} B$ if $B \neq \emptyset$.

(iii) If $A \models^{\alpha} B$ and $\alpha \geq \beta$, then $A \models^{\beta} B$ (nestedness).

(iv) $A \vee B \models{ }^{\alpha} C$ if and only if $A \models{ }^{\alpha} C$ and $B \models{ }^{\alpha} C$ (left OR).

(v) Assume that $C$ is a one-element set. Then $C \models^{\alpha} A \vee B$ if and only if $C=^{\alpha} A$ or $C==^{\alpha} B$ (right $O R$ ).

(vi) Assume that $A$ and $B$ are one-element sets. Then $A={ }^{\alpha} B$ if and only if $B=^{\alpha} A$ (symmetry).

(vii) If $A \models^{\alpha} B$ and $B \models^{\beta} C$ then $A \models{ }^{\alpha \odot \beta} C \quad(\odot$-transitivity).

(viii) If $A \models^{\alpha} B$ and $B \models C$, then $A=^{\alpha} C$ (right weakening).

(ix) If $A \models B$ and $B \models{ }^{\alpha} C$, then $A \models{ }^{\alpha} C$ (left strengthening). 
Indeed, when $W$ is finite, properties (i) to (vii) fully characterise the approximate entailment, as shown in [2] (properties (viii) and (ix) easily follow from (i) and (vii)).

Theorem 2.4. Let $W$ be a non-empty finite set, and let $\left\{\vdash_{\alpha}\right\}_{\alpha \in V}$ be a family of binary relations between subsets of $W$ satisfying the properties of Lemma 2.3 (i.e. with $\vdash_{\alpha}$ replacing all occurrences of $\models^{\alpha}$ ). Then there exists a similarity $S$ on $W$ such that $\vdash_{\alpha}=\models{ }^{\alpha}$, for each $\alpha \in V$.

A logic to reason about conditionals capturing the notion of the graded entailment relations $\models{ }^{\alpha}$ is presented in Section 4 .

\section{A graded strong entailment}

The setting for strong reasoning is the following. We again start with a similarity space $(W, S)$ and we proceed in a way which is, in a sense, complementary to the case of approximate reasoning.

In the case of approximate reasoning, we weakened the classical implicational relationship (3); its graded version is (4). In the present case, (3) is strengthened: the relationship (3) is not only required to hold, but (3) is required to be stable under sufficiently small changes of the arguments.

More precisely, for a set $A \subseteq W$ of worlds, a $w \in W$, and a similarity degree $\alpha \in[0,1]$, we define $w \approx^{\alpha} A$ to hold if $v \models A$ for any $v$ such that $S(v, w) \geq \alpha$. Note that, even if $A \neq \emptyset$, there may not exist any $w \in W$ for which $w \approx^{\alpha} A$ holds for a given $\alpha$. We say that $A$ strongly implies $B$ to the degree $\alpha$ if

$$
\text { for any } w \in W, \quad w \models A \text { implies } w \approx^{\alpha} B \text {. }
$$

We write $A \approx^{\alpha} B$ for (5). Obviously,

$$
A \approx^{\alpha} B \text { if and only if } U_{\alpha}(A) \subseteq B .
$$

Unlike the case of approximate entailment, for a given pair of sets $A, B \subseteq W$, it may well be the case that there does not exist a value $\alpha \in[0,1]$ such that $A \approx^{\alpha} B$. Indeed, this is the case exactly if $A \nsubseteq B$. Furthermore, if such an $\alpha$ exists, that is, if $A \subseteq B$, one might wonder again how to calculate the best value for $\alpha$. To this end, let us consider Ruspini's second measure of the compatibility of one proposition with another one: $C(B \mid A)=\sup _{v \models A, w \models B} S(v, w)$. Consider now

$$
J(B \mid A)=C(\neg B \mid A)=\sup _{v \models A, w \models \neg B} S(v, w) .
$$


Then again, $J(B \mid A)<\alpha$ implies $A \approx^{\alpha} B$. Under certain additional assumptions like for instance the finiteness of $W$, we have again an equivalence.

Proposition 3.1. Assume that $W$ is finite. Let $\alpha>0$. Then $A \approx^{\alpha} B$ if and only if $J(B \mid A)<\alpha$.

Proof. We have $J(B \mid A)=\max _{v \in A, w \notin B} S(v, w)$. Hence $J(B \mid A)<\alpha$ iff, for all $v \in A$ and $w \notin B, S(v, w)<\alpha$ iff, for all $v \in A$ and $w \in W, w \notin B$ implies $S(v, w)<\alpha$ iff, for all $v \in A$ and $w \in W, S(v, w) \geq \alpha$ implies $w \in B$ iff $U_{\alpha}(A) \subseteq B$. The last statement is, by definition, equivalent to $A \approx^{\alpha} B$.

We compile the basic properties of the strong entailment relation.

Lemma 3.2. Let $(W, S)$ be a similarity space. Then the following holds for any $A, B, C \subseteq W$ and $\alpha, \beta \in V$ :

(i) $A \approx^{1} B$ if and only if $A \models B$; in particular $A \approx^{1} A$ (reflexivity).

(ii) $A \approx^{0} B$ if and only if $A=\emptyset$ or $B=W$.

(iii) If $A \approx^{\alpha} B$ and $\alpha \leq \beta$, then $A \approx^{\beta} B$ (nestedness).

(iv) $A \vee C \approx^{\alpha} B$ if and only if $A \approx^{\alpha} B$ and $C \approx^{\alpha} B$ (left $O R$ ).

(v) $C \approx^{\alpha} A \wedge B$ if and only if $C \approx^{\alpha} A$ and $C \approx^{\alpha} B$ (right $A N D$ ).

(vi) If $A \approx^{\alpha} B$, then $\neg B \approx^{\alpha} \neg A$ (contraposition).

(vii) Assume that $A, B, C$ are one-element. If $A \approx^{\alpha \odot \beta} \neg B$, then either $A \approx^{\alpha} \neg C$ or $C \approx^{\beta} \neg B \quad$ (restricted $\odot$-transitivity).

(viii) If $A \approx^{\alpha} B$ and $B \approx^{\beta} C$, then $A \approx^{\min (\alpha, \beta)} C$ (min-transitivity).

(ix) If $A \approx^{\alpha} B$ and $B=C$, then $A \approx^{\alpha} C$ (right weakening).

(x) If $A=C$ and $C \approx^{\alpha} B$, then $A \approx^{\alpha} B$ (left strengthening).

Proof. (i) is an immediate consequence of the properties (sr1) and (sr2) of the similarity relation $S$ which implies the fact that $U_{\alpha}(A)=A$ iff $\alpha=1$.

(ii) is due to the fact that $U_{0}(A)=W$ if $A \neq W$ and $U_{0}(\emptyset)=\emptyset$.

(iii) holds since $U_{\alpha}(A) \subseteq U_{\beta}(A)$ whenever $\alpha \geq \beta$.

(iv) follows from the fact that $U_{\alpha}(A \vee B)=U_{\alpha}(A) \vee U_{\alpha}(B)$ and that $X \vee Y \subseteq Z$ iff both $X \subseteq Z$ and $Y \subseteq Z$. 
(v) Here we use that $X \subseteq Y \wedge Z$ iff both $X \subseteq Y$ and $X \subseteq Z$.

(vi) By definition, $A \approx^{\alpha} B$ iff $U_{\alpha}(A) \subseteq B$, and this happens iff for all $u, v \in W$, if $u \in A$ and $S(u, v) \geq \alpha$ then $v \in B$. But this can be equivalently written as, for all $u, v \in W$, if $u \notin B$ and $S(u, v) \geq \alpha$ then $v \notin A$, and since $S$ is symmetric, this is equivalent to $U_{\alpha}(\neg B) \subseteq \neg A$, and this is just $\neg B \approx^{\alpha} \neg A$.

(vii) Let $A=\{u\}, B=\{v\}$ and $C=\{w\}$. Dropping brackets from singleton sets, we have to show that if $u \approx^{\alpha \odot \beta} \neg v$, then either $u \approx^{\alpha} \neg w$ or $w \approx^{\beta} \neg v$.

Assume that $u \not \varpi^{\alpha} \neg w$ and $w \not \varkappa^{\beta} \neg v$. This means $w \in U_{\alpha}(u)$ and $v \in U_{\beta}(w)$, and it follows $S(u, w) \geq \alpha$ and $S(v, w) \geq \beta$. By ( $\mathrm{sr} 4)$, we conclude $S(u, v) \geq \alpha \odot \beta$. Consequently, $v \in U_{\alpha \odot \beta}(u)$, hence $u \not \varkappa^{\alpha \odot \beta} \neg v$, and the proof is complete.

(viii) Assume $U_{\alpha}(A) \subseteq B$ and $U_{\beta}(B) \subseteq C$. Since $B \subseteq U_{\beta}(B)$, we obviously have $U_{\alpha}(A) \subseteq C$. On the other hand, since $A \subseteq B$, we have $U_{\beta}(A) \subseteq U_{\beta}(B)$, and hence $U_{\beta}(A) \subseteq C$ as well.

(ix) and (x) are easy consequences of (viii) and (i).

Theorem 3.3. Let $W$ be a non-empty finite set, and let $\left\{\vdash^{\alpha}\right\}_{\alpha \in V}$ be a family of binary relations between subsets of $W$ satisfying properties (i)-(vii) of Lemma 3.2 (i.e. with $\vdash^{\alpha}$ replacing all occurrences of $\approx^{\alpha}$ ). Then there exists a similarity $S$ on $W$ such that $\vdash^{\alpha}=\approx^{\alpha}$, for each $\alpha \in V$.

Proof. For each $v, w \in W$, define

$$
S(v, w)=\max \left\{\alpha \in V \mid\{v\} \not \nvdash^{\alpha} \neg\{w\}\right\} .
$$

Note that, by (ii), the set over which the maximum is taken is always non-empty.

By (i), $S$ is reflexive and separating, and by (vi), $S$ is symmetric. Furthermore, it is not difficult to check that, by (vii), $S$ is $\odot$-transitive. Hence $(W, S)$ is a similarity space.

Let now $\approx^{\alpha}$ be defined from $S$ by (6). It remains to show that $\vdash^{\alpha}=\approx^{\alpha}$ for all $\alpha \in V$. So let $A, B \subseteq W$ and $\alpha \in V$. We may write $A=\bigvee_{v \in A}\{v\}$ and $B=\neg\left(\bigvee_{w \in \neg B}\{w\}\right)=\bigwedge_{w \in \neg B} \neg\{w\}$. By (iv) and (v), we conclude that $A \vdash^{\alpha} B$ iff for every $v \in A$ and $w \notin B$ we have $\{v\} \vdash^{\alpha} \neg\{w\}$.

On the other hand, $A \approx^{\alpha} B$ holds iff for every $v \in A$ and every $w \in W, S(v, w) \geq$ $\alpha$ implies $w \in B$. That is, $A \approx^{\alpha} B$ iff for every $v \in A$ and $w \notin B$ we have $S(v, w)<\alpha$. But $\{v\} \vdash^{\alpha} \neg\{w\}$ iff $S(v, w)<\alpha$, and the proof is complete.

A logic to reason about conditionals capturing the notion of the graded entailment relations $\approx^{\alpha}$ is presented in Section 5 . 


\section{A logic of graded approximate entailment}

We will now present a logic to reason about conditional (syntactic) objects capturing the idea of the approximate entailment relations $=^{\alpha}$ in similarity spaces. The work is based on R. Rodríguez' thesis [11], although the logic introduced here differs slightly from the version presented in [11].

Throughout this section, let us fix a t-norm $\odot$ and a set $V$ of similarity degrees. $V$ is required to fulfil the following conditions: (i) $0,1 \in V$; (ii) $V$ is closed under $\odot$; (iii) there is no infinite sequence of strictly increasing elements of $V$. Note that (iii) means that $V$ is a Noetherian subchain of $[0,1] ;{ }^{2}$ in particular, any non-empty subset of $V$ is required to contain a largest element. Furthermore, we will work with a fixed finite set $N \geq 1$ of variables.

We define the Logic of Approximate Entailment, or LAE for short, model-theoretically as follows.

Definition 4.1. The propositional formulas of LAE are built up from a finite set of variables $\varphi_{1}, \ldots, \varphi_{N}$ and the constants $\perp, \top$ by means of the binary operators $\wedge$ and $\vee$ and the unary operator $\neg$. The set of propositional formulas is denoted by $\mathcal{P}$. A graded implication of LAE is a triple consisting of two propositional formulas $\varphi$ and $\psi$ as well as a value $\alpha \in V$, denoted by

$$
\varphi>_{\alpha} \psi
$$

A (general) formula of LAE is built up from the graded implications and the constants $\perp, \top$ by means of the binary operators $\wedge$ and $\vee$ and the unary operator $\neg$.

Let $(W, S)$ be a similarity space. An evaluation for LAE is a mapping $e$ from $\mathcal{P}$ to the power set of $W$ such that, for any $\varphi, \psi \in \mathcal{P}, e(\varphi \wedge \psi)=e(\varphi) \cap e(\psi)$, $e(\varphi \vee \psi)=e(\varphi) \cup e(\psi), e(\neg \varphi)=W \backslash e(\varphi), e(\perp)=\emptyset, e(\top)=W$.

Let $e$ be an evaluation. Then a graded implication $\varphi>_{\alpha} \psi$ is satisfied by $e$ if

$$
e(\varphi) \subseteq U_{\alpha}(e(\psi))
$$

and the satisfaction of the remaining formulas of LAE is defined like in classical propositional logic.

A theory of LAE is a set of formulas. We say that a theory $\mathcal{T}$ semantically entails a formula $\Phi$, written $\mathcal{T} \models \operatorname{LAE} \Phi$, if any evaluation satisfying all elements of $\mathcal{T}$ also satisfies $\Phi$.

\footnotetext{
${ }^{2}$ Thanks are due to an anonymous referee for this comment.
} 
In other words, the propositional formulas of LAE are those of classical propositional logic (CPL for short). Graded implications are implications between two propositional formulas subject to a weight between 0 and 1 , and they are the smallest units of formulas of LAE. Finally, graded implications can be combined in turn by using CPL connectives.

In contrast to [11], we allow only formulas built up from graded implications rather than from CPL formulas as well. This is a restriction of the language; note, however, that the following holds. A CPL formula $\varphi$ can be translated to the LAE formula $T>_{1} \varphi$. Indeed, one can easily show that CPL is included in LAE in the following sense. Denote by $\varphi^{*}$ the LAE formula $T>_{1} \varphi$, and let $\Gamma^{*}=\left\{\varphi^{*}: \varphi \in \Gamma\right\}$. Then, for every finite set $\Gamma$ of propositional formulas, $\Gamma \models_{\mathrm{CPL}} \varphi$ if, and only if, $\Gamma^{*} \models \operatorname{LAE} \varphi^{*}$.

We will use in the sequel the defined implication $\rightarrow$ and equivalence $\leftrightarrow$ connectives as usual in CPL, and we endow them with lower precedence than the remaining connectives. Moreover, by a maximally elementary conjunction, or m.e.c. for short, we mean the conjunction of all variables of LAE each of which can be negated or unnegated.

Definition 4.2. The following are axioms of $\operatorname{LAE}$, for any $\varphi, \psi, \chi \in \mathcal{P}$ and $\alpha, \beta \in$ $V$ :

(A1) $\varphi>_{1} \psi$, where $\varphi, \psi$ are such that $\varphi \rightarrow \psi$ is a tautology of CPL

(A2) $\left(\varphi>_{\alpha} \psi\right) \rightarrow\left(\varphi>_{\beta} \psi\right)$, where $\alpha \geq \beta$

(A3) $\left(\varphi>_{0} \psi\right) \vee(\psi>1 \perp)$

(A4) $\left(\varphi>_{\alpha} \perp\right) \rightarrow\left(\varphi>_{1} \perp\right)$

(A5) $\left(\delta>_{\alpha} \varepsilon\right) \rightarrow\left(\varepsilon>_{\alpha} \delta\right) \vee\left(\delta>_{1} \perp\right)$, where $\delta$ and $\varepsilon$ are m.e.c.'s

(A6) $\left(\varphi>_{\alpha} \chi\right) \wedge\left(\psi>_{\alpha} \chi\right) \rightarrow\left(\varphi \vee \psi>_{\alpha} \chi\right)$

(A7) $\left(\varepsilon>_{\alpha} \varphi \vee \psi\right) \rightarrow\left(\varepsilon>_{\alpha} \varphi\right) \vee\left(\varepsilon>_{\alpha} \psi\right)$, where $\varepsilon$ is a m.e.c.

(A8) $\left(\varphi>_{1} \psi\right) \rightarrow\left(\varphi \wedge \neg \psi>_{1} \perp\right)$

(A9) $\left(\varphi>_{\alpha} \psi\right) \wedge\left(\psi>_{\beta} \chi\right) \rightarrow\left(\varphi>_{\alpha \odot \beta} \chi\right)$

(A10) Given a tautology of CPL, the formula resulting from a uniform replacement of the variables by graded implications of LAE is an axiom of LAE.

Finally, the following is a rule of LAE for any formulas $\Phi, \Psi$ : 
(MP) $\frac{\Phi \quad \Phi \rightarrow \Psi}{\Psi}$

A proof of a formula $\Phi$ from a theory $\mathcal{T}$ is defined as usual. If it exists, we write $\mathcal{T} \vdash_{\text {LAE }} \Phi$. A theory $\mathcal{T}$ is called consistent if $\mathcal{T}$ does not prove $T>_{1} \perp$.

We include a couple of lemmas to simplify the subsequent completeness proof for LAE.

Lemma 4.3. [Substitution of classically equivalent formulas] Let $\varphi, \psi, \chi$ be LAE propositional formulas. If $\varphi \leftrightarrow \psi$ is a CPL tautology, then, for each $\alpha \in V$, LAE proves $\left(\varphi>_{\alpha} \chi\right) \leftrightarrow\left(\psi>_{\alpha} \chi\right)$ and $\left(\chi>_{\alpha} \varphi\right) \leftrightarrow\left(\chi>_{\alpha} \psi\right)$.

Proof. Let $\psi \rightarrow \varphi$ be a CPL tautology. Then, by (A1), LAE proves $\psi>_{1} \varphi$, and by (A9) it also proves $\left(\psi>_{1} \varphi\right) \wedge\left(\varphi>_{\alpha} \chi\right) \rightarrow\left(\begin{array}{lll}\psi>_{\alpha} & \chi\end{array}\right)$; hence LAE proves $\left(\varphi>_{\alpha} \chi\right) \rightarrow\left(\psi>_{\alpha} \chi\right)$. Similarly, we argue in case of the remaining implications.

Lemma 4.4. Let $\varphi, \psi, \chi$ be $\mathrm{LAE}$ propositional formulas. Then $\mathrm{LAE}$ proves the following.

(i) $\left(\varphi \wedge \neg \psi>_{1} \perp\right) \rightarrow\left(\varphi>_{1} \psi\right)$.

(ii) $\left(\varphi>_{1} \psi\right) \wedge\left(\varphi>_{1} \chi\right) \rightarrow\left(\varphi>_{1} \psi \wedge \chi\right)$.

Proof. (i) By (A1) and (A9), we get $\left(\varphi \wedge \neg \psi>_{1} \perp\right) \rightarrow\left(\varphi \wedge \neg \psi>_{1} \psi\right)$. By (A1) we have $\varphi \wedge \psi>_{1} \psi$. Hence $\left(\varphi \wedge \neg \psi>_{1} \perp\right) \rightarrow\left((\varphi \wedge \neg \psi) \vee(\varphi \wedge \psi)>_{1} \psi\right)$, and the claim follows.

(ii) By (A8), we derive $\left(\varphi>_{1} \psi\right) \rightarrow\left(\varphi \wedge \neg \psi>_{1} \perp\right)$ and $\left(\varphi>_{1} \chi\right) \rightarrow\left(\varphi \wedge \neg \chi>_{1}\right.$ $\perp)$. By (A6), we get $\left(\varphi>_{1} \psi\right) \wedge\left(\varphi>_{1} \chi\right) \rightarrow\left((\varphi \wedge \neg \psi) \vee(\varphi \wedge \neg \chi)>_{1} \perp\right)$ and hence $\left(\varphi>_{1} \psi\right) \wedge\left(\varphi>_{1} \chi\right) \rightarrow\left(\varphi \wedge \neg(\psi \wedge \chi)>_{1} \perp\right)$. By part (i), the claim follows.

Theorem 4.5. Let $\mathcal{T}$ be a consistent theory and $\Phi$ be a formula of LAE. Then $\mathcal{T} \vdash_{\mathrm{LAE}} \Phi$ if and only if $\mathcal{T} \models \operatorname{LAE} \Phi$.

Proof. In view of Lemma 2.3, axioms (A1)-(A4) and (A6)-(A9) are valid. Furthermore, let $\delta$ and $\varepsilon$ be m.e.c.'s. If then $\delta$ and $\varepsilon$ are modelled by non-empty subsets, these subsets are either disjoint or coincide. Thus, if $\delta>_{\alpha} \varepsilon$ is satisfied, either $\delta$ is modelled by the empty set or otherwise $\delta$ and $\varepsilon$ are modelled by the same sets. It follows that (A5) is valid as well. The validity of (A10) and the soundness of (MP) are clear. Thus $\mathcal{T} \vdash_{\text {LAE }} \Phi$ implies $\mathcal{T} \models_{\text {LAE }} \Phi$. 
Assume that $\mathcal{T}$ does not prove $\Phi$. Let $\overline{\mathcal{T}}$ be a consistent theory such that $\mathcal{T} \subseteq \overline{\mathcal{T}}$, $\Phi \notin \overline{\mathcal{T}}$, and for any formula $\Psi$, either $\Psi \in \overline{\mathcal{T}}$ or $\neg \Psi \in \overline{\mathcal{T}}$. Such a theory exists; using (A10), we show this in the same way as in CPL.

For $\varphi, \psi \in \mathcal{P}$, put $\varphi \approx \psi$ if $\overline{\mathcal{T}} \vdash \varphi>_{1} \psi$ and $\overline{\mathcal{T}} \vdash \psi>_{1} \varphi$. Then $\approx$ is clearly an equivalence relation. Using Lemma 4.4(ii), it is easily checked that $\approx$ is compatible with $\wedge$. By $(\mathrm{A} 6), \approx$ is also compatible with $\vee$. By Lemma 4.4(i), we see that $\approx$ is also compatible with $\neg$. Let $([\mathcal{P}] ; \wedge, \vee, \neg,[\perp],[\top])$ be the induced algebra.

Then $[\mathcal{P}]$ is a Boolean algebra. In fact, for any formulas $\varphi, \psi \in \mathcal{P}$ which are equivalent in CPL, we have $\varphi \approx \psi$ by (A1) and thus $[\varphi]=[\psi]$, and it follows that any equality holding in all Boolean algebras holds in $[\mathcal{P}]$. Note that $[\varphi] \leq[\psi]$ iff $[\varphi] \wedge[\psi]=[\varphi]$ iff $\varphi \wedge \psi \approx \varphi$ iff $\varphi>_{1} \psi$.

Let $W$ be the set of atoms of $[\mathcal{P}] . W$ is nonempty by the consistency of $\overline{\mathcal{T}}$. For any $[\delta],[\varepsilon] \in W$, we define

$$
S([\delta],[\varepsilon])=\max \left\{\alpha \in V: \overline{\mathcal{T}} \vdash \delta>_{\alpha} \varepsilon\right\} .
$$

Note that $\overline{\mathcal{T}} \vdash \delta>_{0} \varepsilon$ by (A3) so that the set over which the maximum is taken is nonempty, and that the maximum of any non-empty subset of $V$ exists by assumption. $S$ fulfils (sr1) by (A1). Furthermore, $\overline{\mathcal{T}} \vdash \delta>_{1} \varepsilon$ implies by (A8) that $[\delta] \wedge \neg[\varepsilon]=[\perp]$, so that $[\delta]$ and $[\varepsilon]$ cannot be distinct atoms of $[\mathcal{P}]$; thus (sr2) holds as well. (sr3) holds by (A5) and (sr4) holds by (A9). It follows that $S$ is a similarity relation w.r.t. $\odot$.

Identifying $[\varphi] \in[\mathcal{P}]$ with the set of atoms below $[\varphi]$, we define the neighbourhood operator $U_{\alpha}:[\mathcal{P}] \rightarrow[\mathcal{P}]$ for each $\alpha \in V$.

Let $\varphi, \psi \in \mathcal{P}$ and $\alpha \in V$; we claim that $\overline{\mathcal{T}} \vdash \varphi>_{\alpha} \psi$ if and only if $[\varphi] \leq U_{\alpha}([\psi])$. If $[\varphi]=[\perp]$, this is evident. If $[\psi]=[\perp]$, both statements imply that $[\varphi]=[\perp]$. So assume that $[\varphi]$ and $[\psi]$ are both non-zero. By (A6), $\overline{\mathcal{T}} \vdash \varphi>_{\alpha} \psi$ holds iff $\overline{\mathcal{T}} \vdash \delta>_{\alpha} \psi$ for all atoms $[\delta] \leq[\varphi]$. By (A7) and the completeness of $\overline{\mathcal{T}}$, for any atom $[\delta], \overline{\mathcal{T}} \vdash \delta>_{\alpha} \psi$ iff $\overline{\mathcal{T}} \vdash \delta>_{\alpha} \varepsilon$ for some atom $[\varepsilon] \leq[\psi]$. For atoms $[\delta],[\varepsilon] \in W, \overline{\mathcal{T}} \vdash \delta>_{\alpha} \varepsilon$ holds iff $S([\delta],[\varepsilon]) \geq \alpha$. Putting these facts together, we see that $\overline{\mathcal{T}} \vdash \varphi>_{\alpha} \psi$ iff for all atoms $[\delta] \leq[\varphi]$ there is an atom $[\varepsilon] \leq[\psi]$ such that $S([\delta],[\varepsilon]) \geq \alpha$, that is, iff $[\varphi] \leq U_{\alpha}([\psi])$.

Let $e: \mathcal{P} \rightarrow[\mathcal{P}], \varphi \mapsto[\varphi]$; then $e$ is an evaluation for LAE. Let $\Psi$ be a graded implication. By what we have just proved, $\overline{\mathcal{T}} \vdash_{\mathrm{LAE}} \Psi$ if and only if $\Psi$ is satisfied by $e$. Let $\Psi$ now an arbitrary formula of LAE. By induction over the complexity of $\Psi$, we conclude that, still, $\overline{\mathcal{T}} \vdash_{\mathrm{LAE}} \Psi$ if and only if $\Psi$ is satisfied by $e$. In particular, all elements of $\mathcal{T}$ are satisfied by $e$, but $\Phi$ is not. 


\section{A logic of graded strong entailment}

In this section we will turn to the question how to axiomatise reasoning about the strong entailment relations $\approx^{\alpha}$ in similarity spaces.

We fix a t-norm $\odot$ and a set $V \subseteq[0,1]$ of similarity degrees according to the same criteria as in the last section. We furthermore fix $N \geq 1$.

We define the Logic of Strong Entailment, or LSE for short, model-theoretically as follows.

Definition 5.1. The propositional formulas of LSE are built up from a finite set of variables $\varphi_{1}, \ldots, \varphi_{N}$ and the constants $\perp, \top$ by means of the binary operators $\wedge$ and $\vee$ and the unary operator $\neg$. The set of propositional formulas is denoted by $\mathcal{P}$. A graded implication of LSE is a triple consisting of two propositional formulas $\varphi$ and $\psi$ as well as a value $\alpha \in V$, denoted by

$$
\varphi \succ_{\alpha} \psi
$$

A (general) formula of LSE is built up from graded implications and the constants $\perp, T$ by means of the binary operators $\wedge$ and $\vee$ and the unary operator $\neg$.

Let $(W, S)$ be a similarity space. An evaluation for LSE is a mapping $e$ from $\mathcal{P}$ to the power set of $W$ similarly to the case of LAE. Let $e$ be an evaluation. Then a graded implication $\varphi \succ_{\alpha} \psi$ is satisfied by $e$ if

$$
U_{\alpha}(e(\varphi)) \subseteq e(\psi)
$$

and the satisfaction of the remaining formulas of LSE is defined like in CPL.

A theory of LSE is a set of formulas. We say that a theory $\mathcal{T}$ semantically entails a formula $\Phi$, written $\mathcal{T} \models{ }_{L S E} \Phi$, if all evaluations satisfying all elements of $\mathcal{T}$ also satisfy $\Phi$.

So the language of LSE shares the two-level concept of LAE, and the only difference is that weighted implications are now understood to strengthen the relationship rather than to weaken it.

Definition 5.2. The following are axioms of LSE, for any $\varphi, \psi, \chi \in \mathcal{P}$ and $\alpha, \beta \in$ $V$ :

(S1) $\varphi \succ_{1} \psi$, where $\varphi, \psi$ are such that $\varphi \rightarrow \psi$ is a tautology of CPL

(S2) $\perp \succ_{0} \varphi$ 
(S3) $\varphi \succ_{0} \top$

(S4) $\left(\varphi \succ_{0} \psi\right) \rightarrow\left(\varphi \succ_{1} \perp\right) \vee\left(\top \succ_{1} \psi\right)$

(S5) $\left(\varphi \succ_{\alpha} \psi\right) \rightarrow\left(\varphi \succ_{\beta} \psi\right)$, where $\alpha \leq \beta$

(S6) $\left(\varphi \succ_{\alpha} \psi\right) \wedge\left(\varphi \succ_{\alpha} \chi\right) \rightarrow\left(\varphi \succ_{\alpha} \psi \wedge \chi\right)$

(S7) $\left(\varphi \succ_{\alpha} \chi\right) \wedge\left(\psi \succ_{\alpha} \chi\right) \rightarrow\left(\varphi \vee \psi \succ_{\alpha} \chi\right)$

(S8) $\left(\varphi \succ_{\alpha} \psi\right) \rightarrow\left(\neg \psi \succ_{\alpha} \neg \varphi\right)$

(S9) $\left(\varphi \succ_{\alpha} \psi\right) \wedge\left(\psi \succ_{\beta} \chi\right) \rightarrow\left(\varphi \succ_{\min \{\alpha, \beta\}} \chi\right)$

(S10) $\left(\varphi \succ_{\alpha \odot \beta} \psi\right) \rightarrow\left(\varepsilon \succ_{\alpha} \neg \varphi\right) \vee\left(\varepsilon \succ_{\beta} \psi\right)$, where $\varepsilon$ is a m.e.c.

(S11) Given a tautology of CPL, the statement resulting from a uniform replacement of the atoms by graded implications of LSE is an axiom.

Furthermore, (MP) is the only rule of inference of LSE (cf. Definition 4.1).

A proof of a formula $\Phi$ from a theory $\mathcal{T}$ is defined as usual. If it exists, we write $\mathcal{T} \vdash_{\text {LSE }} \Phi$. A theory $\mathcal{T}$ is called consistent if $\mathcal{T}$ does not prove $\top \succ_{1} \perp$.

Theorem 5.3. Let $\mathcal{T}$ be a consistent theory and $\Phi$ be a formula of LSE. Then $\mathcal{T} \vdash_{\mathrm{LSE}} \Phi$ if and only if $\mathcal{T} \models$ LSE $\Phi$.

Proof. By Lemma 3.2, axioms (S1)-(S10) are valid. Clearly, also (S11) is valid and (MP) is sound. It follows that $\mathcal{T} \vdash$ LSE $\Phi$ implies $\mathcal{T} \models$ LSE $\Phi$.

Assume that $\mathcal{T}$ does not prove $\Phi$. We proceed like in the proof of Theorem 4.5 to construct $\overline{\mathcal{T}}$. Furthermore, we define the equivalence relation $\approx$ by putting $\varphi \approx \psi$ if $\overline{\mathcal{T}} \vdash \varphi \succ_{1} \psi$ and $\overline{\mathcal{T}} \vdash \psi \succ_{1} \varphi$, for $\varphi, \psi \in \mathcal{P}$. [P $]$ will be the set of equivalence classes.

It is not difficult to check that $\approx$ is compatible with $\wedge, \vee$, and $\neg$. Like in the proof of Theorem 4.5 , we conclude that the quotient algebra $([\mathcal{P}] ; \wedge, \vee, \neg,[\perp],[\top])$ is a Boolean algebra.

Let $W$ be the set of atoms of $[\mathcal{P}]$. Again, $W$ is nonempty by the consistency of $\overline{\mathcal{T}}$. For any $[\delta],[\varepsilon] \in W$, we define

$$
S([\delta],[\varepsilon])=\max \left\{\alpha: \overline{\mathcal{T}} \nvdash \delta \succ_{\alpha} \neg \varepsilon\right\} .
$$

Note that the set over which the maximum is taken is non-empty. Indeed, $\overline{\mathcal{T}} \vdash$ $\delta \succ_{0} \neg \varepsilon$ would imply by (S4) that $[\delta]$ or $[\varepsilon]$ equals $[\perp]$. Note furthermore that the maximum exists by assumption. 
We remark that for any $[\delta],[\varepsilon] \in W$ and $\alpha \in V$, we have $\overline{\mathcal{T}} \vdash \delta \succ_{\alpha} \neg \varepsilon$ iff $S([\delta],[\varepsilon])<\alpha$.

$S$ fulfils (sr1). Indeed, assume the contrary; then there is an atom $[\varepsilon] \in W$ such that $\overline{\mathcal{T}} \vdash \varepsilon \succ_{1} \neg \varepsilon$ so that, in view of (S6), $\overline{\mathcal{T}} \vdash \varepsilon \succ_{1} \perp$ although $[\varepsilon]$ is non-zero. Furthermore, $S$ fulfils (sr2). Indeed, let $[\delta],[\varepsilon] \in W$ be distinct; then $\overline{\mathcal{T}} \vdash \delta \wedge \varepsilon \succ_{1}$ $\perp$ so that, in view of (S7), $\overline{\mathcal{T}} \vdash(\delta \wedge \varepsilon) \vee(\delta \wedge \neg \varepsilon) \succ_{1} \delta \wedge \neg \varepsilon$, hence $\overline{\mathcal{T}} \vdash \delta \succ_{1} \neg \varepsilon$ and $S([\delta],[\varepsilon])<1$. Furthermore, $S$ fulfils (sr3) by (S8).

Finally, $S$ is also $\odot$-transitive. To see this, let $[\delta],[\varepsilon],[\zeta] \in W$ and let $\alpha=$ $S([\delta],[\varepsilon])$ and $\beta=S([\varepsilon],[\zeta])$. Then $\overline{\mathcal{T}} \nvdash \varepsilon \succ_{\alpha} \neg \delta$ and $\overline{\mathcal{T}} \nvdash \varepsilon \succ_{\beta} \neg \zeta$. By the completeness of $\overline{\mathcal{T}}$, this means $\overline{\mathcal{T}} \vdash \neg\left(\varepsilon \succ_{\alpha} \neg \delta\right) \wedge \neg\left(\varepsilon \succ_{\beta} \neg \zeta\right)$. It follows by (S10) that $\overline{\mathcal{T}} \vdash \neg\left(\delta \succ_{\alpha \odot \beta} \neg \zeta\right)$. Hence $\overline{\mathcal{T}} \nvdash \delta \succ_{\alpha \odot \beta} \neg \zeta$ and consequently $S([\delta],[\zeta]) \geq \alpha \odot \beta$. The proof of (sr4) is complete.

Thus $S$ is a $\odot$-similarity relation. Identifying $[\varphi] \in[\mathcal{P}]$ with the set of atoms below $[\varphi]$, we again define the neighbourhood operator $U_{\alpha}$ on $[\mathcal{P}]$ for each $\alpha$.

Let $\varphi, \psi \in \mathcal{P}$; we claim that $\overline{\mathcal{T}} \vdash \varphi \succ_{\alpha} \psi$ if and only if $U_{\alpha}([\varphi]) \subseteq[\psi]$. If $[\varphi]=$ $[\perp]$, this is evident. If $[\psi]=[\perp]$, both statements imply that $[\varphi]=[\perp]$. So assume that $[\varphi]$ and $[\zeta]$ are both non-zero. Then $[\varphi]=\bigvee_{[\delta] \leq[\varphi]}[\delta]$ and $[\psi]=\bigwedge_{[\varepsilon] \leq \neg[\psi]}[\neg \varepsilon]$, where $[\delta],[\varepsilon]$ are understood to run over the atoms. By (S6) and (S7), $\mathcal{T} \vdash \varphi \succ_{\alpha} \psi$ holds iff $\overline{\mathcal{T}} \vdash \delta \succ_{\alpha} \neg \varepsilon$ for all $[\delta] \leq[\varphi]$ and $[\varepsilon] \leq \neg[\psi]$, that is, iff $S([\delta],[\varepsilon])<\alpha$ for all $[\delta] \leq[\varphi]$ and $[\varepsilon] \leq \neg[\psi]$. Furthermore, $U_{\alpha}([\varphi]) \subseteq[\psi]$ holds iff, for all $[\delta] \leq[\varphi]$ and all $[\varepsilon]$ such that $S([\delta],[\varepsilon]) \geq \alpha,[\varepsilon] \leq[\psi]$. The claim is proved.

Thus $(W, S)$ is a similarity space in which all elements of $\mathcal{T}$ are satisfied by the natural evaluation $\varphi \mapsto[\varphi]$, but, in view of the completeness of $\overline{\mathcal{T}}$, it does not satisfy $\Phi$.

\section{Concluding remarks}

In this paper we have discussed two logics based on different types of conditionals, extending the possibilities of classical propositional logic to express logical relationships between propositions. Namely, a similarity relation on the space of propositions is assumed and integrated into the calculus. In case of the Logic of Approximate Entailment, implicational relationships are treated in a relaxed way; deviations from a correct implication are allowed to a given extent. In case of the Logic of Strong Entailment, implicational relationships are treated in a strict way; deviations to a given extent must not affect the correctness of an implication.

There are many ways to further develop this work. An obvious one is to combine 
the two logics such that one could reason about both kinds of (graded) implications, the relaxed ones $>_{\alpha}$ and the strict ones $\succ_{\alpha}$. For instance, given the semantics based on similarity spaces we have introduced for the two graded approximate and strong implications, one should be able to conclude that $\psi$ (classically) follows from $\varphi$ from the two premises $\varphi>_{\alpha} \chi$ and $\chi \succ_{\alpha} \psi$.

The resulting Logic for Approximate and Strong Entailments, or LASE for short, can easily be defined as follows. The propositional formulas of LASE are defined in the same way as in the LAE and LSE logics. A (general) formula of LASE is built up from graded LAE-implications $\varphi>_{\alpha} \psi$, LSE-implications $\varphi \succ_{\alpha} \psi$ and the constants $\perp$ and $T$, by means of the binary operators $\wedge$ and $\vee$ and the unary operator $\neg$. Given a similarity space $(W, S)$, an evaluation for LASE is again a mapping $e$ from $\mathcal{P}$ to the power set of $W$, and the satisfaction of graded LAE-implications and LSE-implications is defined exactly as in LAE and in LSE respectively. In the same way, given a theory $\mathcal{T}$ over LASE and a LASE-formula $\Phi$, one can define $\mathcal{T} \models$ LASE $\Phi$ when all evaluations satisfying all elements of $\mathcal{T}$ also satisfy $\Phi$.

An axiomatization (possibly not minimal) of LASE can be achieved by gathering all axioms of LAE and LSE, plus the following four additional ones relating the two kinds of conditionals:

(AS1) $\left(\varphi>_{1} \psi\right) \rightarrow\left(\varphi \succ_{1} \psi\right)$

(AS2) $\left(\varphi>_{\alpha} \psi\right) \wedge\left(\psi \succ_{\alpha} \chi\right) \rightarrow\left(\varphi>_{1} \chi\right)$

(AS3) $\left(\varepsilon>_{\alpha} \delta\right) \leftrightarrow \neg\left(\delta \succ_{\alpha} \neg \varepsilon\right)$, where $\varepsilon, \delta$ are m.e.c.'s

(AS4) Given a tautology of CPL, the statement resulting from a uniform replacement of the atoms by graded LAE-implications or graded LSE-implications is an axiom.

Modus Ponens remains as the only rule of inference of LASE. The notion of proof in LASE, denoted $\vdash_{\text {LASE }}$, is defined as usual. Note that from (AS2) it follows that $\left(\varphi \succ_{1} \psi\right) \rightarrow\left(\varphi>_{1} \psi\right)$ is a theorem of LASE, hence using (AS1), $\left(\varphi>_{1} \psi\right) \leftrightarrow$ $\left(\varphi \succ_{1} \psi\right)$ is a theorem as well. LASE can also be shown to be also complete with respect to the above defined semantics in terms of similarity spaces.

Theorem 6.1. Let $\mathcal{T}$ be a consistent theory and $\Phi$ be a formula of LASE. Then $\mathcal{T} \vdash_{\text {LASE }} \Phi$ if and only if $\mathcal{T} \models$ LASE $\Phi$.

The proof goes along the same lines as for LAE and LSE. It is worth noticing that, due to axiom (AS1), $\overline{\mathcal{T}} \vdash\left(\varphi \succ_{1} \psi\right) \wedge\left(\psi \succ_{1} \varphi\right)$ iff $\overline{\mathcal{T}} \vdash\left(\varphi>_{1} \psi\right) \wedge\left(\psi>_{1} \varphi\right)$, 
so the equivalence relation $\approx$ can be equivalently defined using either $\succ_{1}$ or $>_{1}$. Moreover, due axiom (AS3), the similarity relation $S$ on the set of atoms of $[\mathcal{P}]$ can also be equivalently defined either like in the completeness proof of LAE or as in the completeness proof of LSE.

Another goal of future work is to modify the two logics LAE and LSE such that the language is even further restricted than here, in the sense of calculi purely based on graded implications of the form $\varphi>_{\alpha} \psi$ or $\varphi \succ_{\alpha} \psi$. Finally, it would be desirable to improve the axiom systems which we have presented in a way that finiteness is not an essential requirement to prove completeness; in particular the axioms based on m.e.c.'s could be revised.

Furthermore, the practical applicability of the logics can be checked. The examples in the introduction give just a raw hint how the logics could be applied. For instance a systematic examination how to use LAE and LSE in decision support systems could be interesting.

Finally, links to related approaches should be explored. At least there are three related approaches which seem worth to be compared with LSE:

1. In [1], inspired in the theory of Mathematical Morphology, the authors introduce the notion of erosion of a proposition $B$ in a degree $\alpha$; w.r.t. our notation, they define $E^{\alpha}(B)=\left\{w \in W \mid w \approx^{\alpha} B\right\}=\left\{w \in W \mid U_{\alpha}(w) \subseteq B\right\}$. We note that this notion has also some resemblance to that of the lower approximation $B_{*}$ of $B$ in rough set theory.

2. Also it could be interesting to relate LSE to the notion of a supermodel given in [5] where the authors use this concept in order to present a entailment more robust than the classical one.

3. In [9] and [10] similar ideas are developed, but with different motivations. In some situations, it might be relevant to find the most pertinent worlds that are models of a proposition $B$. This problem is approached in both papers by choosing as most pertinent those $B$-worlds which are least similar to $\neg B$ worlds.

\section{Acknowledgements}

The authors acknowledge partial support of the bilateral Austrian-Spanish project HA2008-0017 and the Eurocores-LogICCC ESF project LoMoReVI. Esteva and Godo also acknowledge partial support of the Spanish project FFI2008-03126E/FILO and Rodríguez acknowledges the projects CyT-UBA X484 and the re- 
search CONICET program PIP 12-200801-02543 2009-2011. Finally, Esteva, Godo and Rodríguez also acknowledge partial support of the MaToMUVI project (PIRSES-GA-2009- 247584). The authors are furthermore grateful to anonymous reviewers for their helpful comments and suggestions.

\section{References}

[1] I. Bloch, J. Lang. Towards mathematical "morpho-logics", in: Technologies for Constructing Intelligent Systems, Vol. 2, 367-380, Springer-Verlag, 2002.

[2] D. Dubois, H. Prade, F. Esteva, P. Garcia, L. Godo. A logical approach to interpolation based on similarity relations, Int. J. Approx. Reasoning 17 (1997), $1-36$.

[3] F. Esteva, P. Garcia, L. Godo, R. O. Rodríguez. A modal account of similaritybased reasoning, Int. J. Approx. Reasoning 16 (1997), 235 - 260.

[4] F. Esteva, L. Godo, R. O. Rodríguez, T. Vetterlein. On the logics of similaritybased approximate and strong entailment, Proc. of the 15th Spanish Congress on Fuzzy Logic and Technology, ESTYLF 2010, Huelva, 187 - 192.

[5] M.L. Ginsberg, A.J. Parkes, A. Roy. Supermodels and robustness. Proc. of the Fifteenth National Conference on Artificial Intelligence, AAAI'98, Madison, Wiconsin, July 1998, 334-339.

[6] L. Godo, R. O. Rodríguez. Logical approaches to fuzzy similarity-based reasoning: an overview, in: G. Della Riccia et al. (eds.). Preferences and similarities, CISM Courses and Lectures 504, Springer-Verlag, Berlin 2008; pp. $75-128$.

[7] O. Kutz. Notes on Logics of Metric Spaces. Studia Logica 85 (2007), 75-104.

[8] O. Kutz, H. Sturm, N.-Y. Suzuki, F. Wolter, and M. Zakharyaschev. Logics of Metric Spaces. ACM Transactions on Computational Logic 4(2), 260-294, 2003.

[9] C. Lafage, J. Lang. Logical representation of preferences for group decision making. In Proc. of the 7th International Conference on Knowledge Representation and Reasoning, KR 2000, pp. 457 - 468, 2000.

[10] R. Pino-Pérez, C.Uzcátegui. Jumping to Explanations versus Jumping to Conclusions. Artificial Intelligence 111, 131-169, 1999. 
[11] R. O. Rodríguez. Aspectos formales en el Razonamiento basado en Relaciones de Similitud Borrosas, Ph. D. Thesis, Technical University of Catalonia (UPC), 2002.

[12] E. H. Ruspini. On the semantics of fuzzy logic, International Journal of Approximate Reasoning 5 (1991), 45 - 88.

[13] M. Ying. A logic for approximate reasoning, J. Symb. Log. 59 (1994), 830 837. 